\title{
SOME ASPECTS OF GALACTIC STRUCTURE DERIVED FROM THE BERKELEY LOW LATITUDE SURVEY OF NEUTRAL HYDROGEN
}

\author{
HAROLD WEAVER \\ University of California, Berkeley, Calif., U.S.A.
}

\begin{abstract}
The principal problem in the study of spiral structure is to reduce the data to a small enough number of pictures or numbers that can be easily comprehended. A new map has been produced, based on circular rotation, but the high probability of large radial motions makes it likely than any picture of spiral structure derived on current theories will be in error. New computer pictures have made it possible to see the structure of the outer arm, which has many cloud-like structures lying above it on one side, some reaching heights of more than $1 \mathrm{kpc}$. Some very weak extended features in the outer regions are described, and examples of holes and jets are discussed.
\end{abstract}

\section{Some Statistics of the Berkeley Low Latitude Survey of Neutral Hydrogen}

Table I displays the statistics of the Berkeley Low Latitude Survey of Neutral Hydrogen. The observational data have been published in the form of profiles (Weaver and Williams, 1973). Contour maps derived from these data in the form $T_{A}(v, b \mid l)$ are in press. The data are also available on magnetic tapes.

An extension of the latitude coverage of the original survey was made to permit investigation of spiral structure at large $z$ distances. The statistics of this secondary program are also shown in Table I. In the form of profiles and contour maps the data of the extended program are in press.

TABLE I

Observational data relating to the Berkeley Low-Latitude Survey of Neutral Hydrogen

Sky coverage:

Primary program:

Every 0.25 in galactic latitude between limits $-10^{\circ}$ to $+10^{\circ}$; every 0.5 in galactic longitude over range $10^{\circ}$ to $250^{\circ}$ (38961 profiles).

Extension program:

Every 0.5 in galactic latitude between limits $-10^{\circ}$ to $-30^{\circ},+10^{\circ}$ to $+30^{\circ}$; every 2.5 in galactic longitude starting at $l=12^{\circ}$, extending to $249^{\circ} .5$.

Frequency coverage:

Frequency resolution:

Integration time:

Rms fluctuations:

Angular resolution:
Each profile covers $250 \mathrm{~km} \mathrm{~s}^{-1} ; 200$ information points per profile, spaced every $5 \mathrm{kHz}$ except at end points of profile.

Information channels $10 \mathrm{kHz}\left(2.11 \mathrm{~km} \mathrm{~s}^{-1}\right)$ wide between half-power points.

$72 \mathrm{~s}$ per profile information point

$0.38 \mathrm{~K}$ (system noise $\leqslant 160 \mathrm{~K}$ )

H.P.B.W. 35.5 


\section{Examples of Use of the Data for Studies of Galactic Structure}

(a) SPIRAL STRUCTURE

The principal problem in the study of spiral structure is to compress the data sufficiently to produce a small number of pictures or descriptive numerical quantities that can be readily comprehended. The basis of the compression technique employed here is illustrated in Figure 1. If neutral hydrogen is distributed in space in spiral form as illustrated in Figure 1a, and if the motion of the gas is governed by a rotation curve monotonically decreasing with increasing radius, we will observe the longitude, velocity diagram shown in Figure 1b. Corresponding points on the loci in the spatial $(R, \theta)$ and observational $(l, v)$ coordinate systems are identified.

It has been pointed out that the picture of high-contrast gas arms is oversimplified. Velocity perturbations arising from gravitational or other effects can produce the observed velocity-antenna temperature structures. Clearly, however, whatever the cause of what is observed, the $l, v$ map provides the fundamental basis for displaying the observational data and delineating the large-scale structure of the system. Our principal task is to find a way to produce the most appropriate and useful $l, v$ map or maps from the observational data.

Various options have been built onto the computer program used to produce $l, v$ maps from the data of the Berkeley Low Latitude Survey. (a) We can specify the range of antenna temperatures from which the map will be made. For example, we may wish to map only that gas that produces an antenna temperature $T_{\mathrm{A}}$ greater than some cutoff value $T_{c}=50 \mathrm{~K}$, say. In this case we map only the most intense features of the observational distribution of hydrogen in the Galaxy. (b) We can map at any latitude or we can take integrals over any specified latitude range and use these integrals in the mapping process. Such integral provides an $l, v$ map which represents a

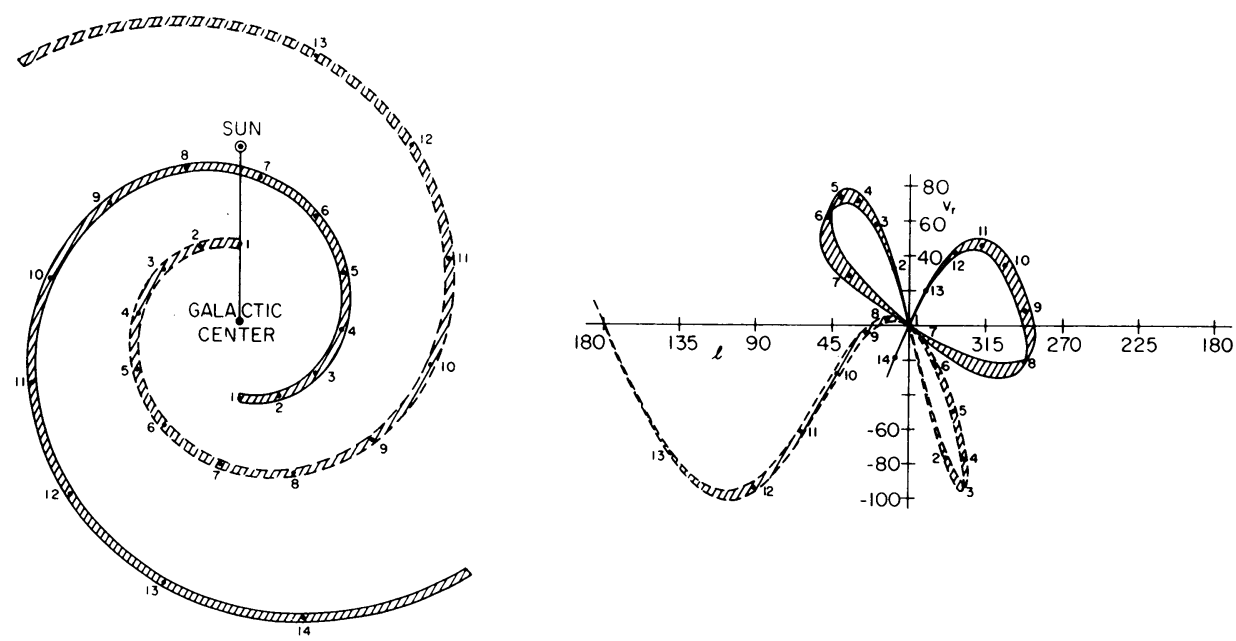

Fig. 1a. Distribution of optically thin neutral hydrogen gas in a model spiral pattern.

Fig. 1b. Observed velocity-longitude diagram for gas distributed as shown in Figure la. 
quasi surface distribution of the gas projected onto the plane of the Galaxy. The basis of this statement is as follows. The function

$$
I\left(v \mid l, T_{\mathrm{A}} \geqslant T_{\mathrm{c}}\right)=\int_{-10^{\circ}}^{+10^{\circ}} T_{\mathrm{A}}\left(v, b \mid l, T_{\mathrm{A}} \geqslant T_{\mathrm{c}}\right) \mathrm{d} b
$$

measures the integrated hydrogen brightness derived by summation over latitude for a given $l, v$ column. If we take velocity as a measure of distance, $r$, in the line of sight, an $l, v$ column is analogous to an $l, r$ column. Summation over $b$ is analogous to summation over $z$, hence $I\left(v \mid l, T_{\mathrm{A}} \geqslant T_{\mathrm{c}}\right)$ represents quasi surface brightness of hydrogen found by projecting onto the galactic plane (that is, summing over $z$ ) all that hydrogen having a given $l, r$ value.

An illustration of $l, v$ map production follows. Figure $2 \mathrm{a}$ shows a characteristic contour map made from the Berkeley data. We specify $T_{\mathrm{c}}=50 \mathrm{~K}$, eliminating all lower antenna temperatures as shown in Figure $2 b$. The contour map in Figure $2 b$ is integrated over $b$ to form $I\left(v, l \mid T_{\mathrm{A}} \geqslant 50 \mathrm{~K}\right)$ illustrated in Figure $2 \mathrm{c}$. The distributions $I\left(v, l \mid T_{\mathrm{A}}>50 \mathrm{~K}\right)$, one for each longitude, are combined to produce the $l, v$ distribution map shown in Figure 3. In these $l, v$ maps the value of $T\left(v, l \mid T_{\mathrm{A}}>T_{\mathrm{c}}\right)$ is represented by blackness. Derived in a similar way, Figure 4 shows the $l, v$ map for $T_{\mathrm{c}}=25 \mathrm{~K}$, while Figure 5 shows the $l, v$ map for $T_{\mathrm{c}}=10 \mathrm{~K}$.

Whatever the physical cause - velocity crowding or arm, interarm hydrogen gas contrast - the general similarity of the observational $l, v$ diagram to the model calculation, Figure 1b, is striking. The sinusoidal loci characteristic of outer spiral features are clearly shown, as is the loop structure of the next inner spiral arm. The observational picture is, of course, more complicated than the smooth theoretical one. Observationally there are many bifurcated features and interarm spurs. The observed

a

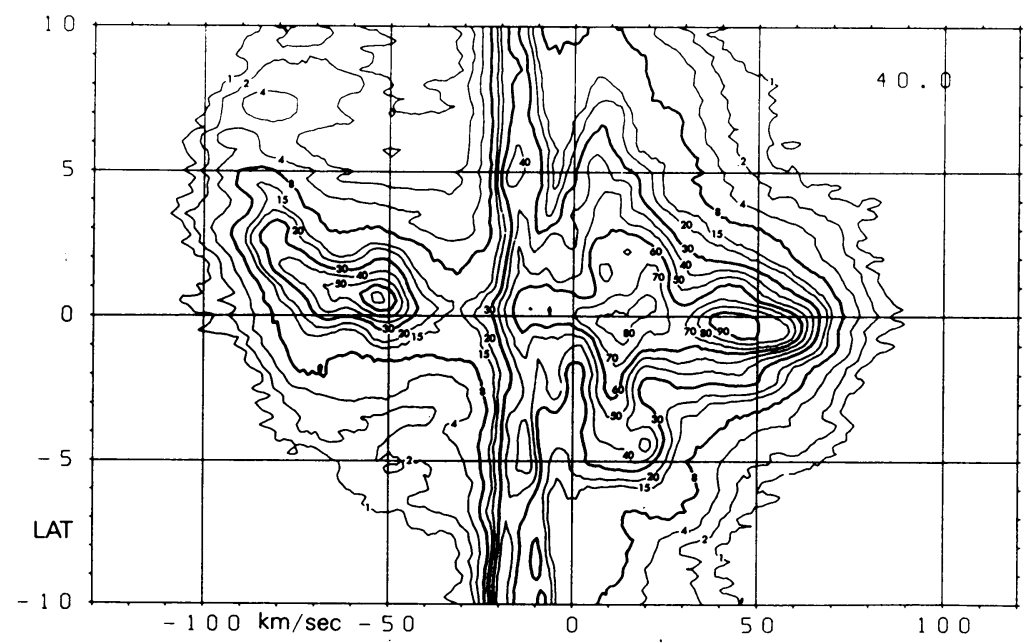

Fig. 2a. Sample contour map derived from the Berkeley Low Latitude Survey of Neutral Hydrogen; $l=40^{\circ}$. 


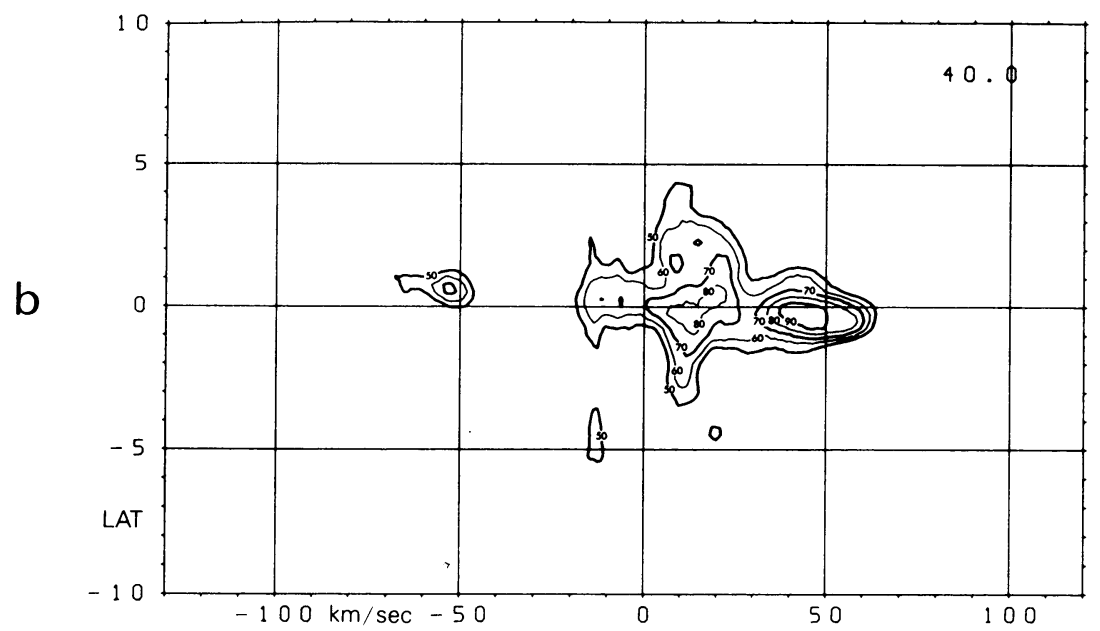

Fig. 2b. Contour map for $T_{\mathrm{A}} \geqslant T_{\mathrm{c}}=50 \mathrm{~K}$ for $l=40^{\circ}$.

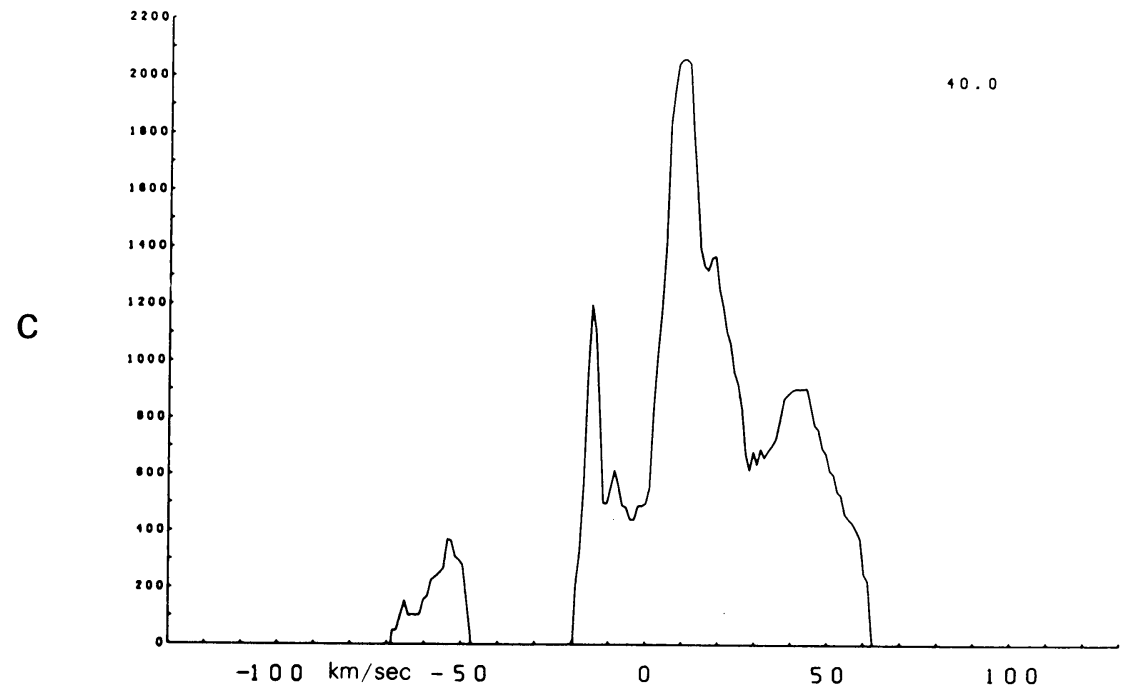

Fig. 2c. The integral $I\left(v \mid l=40^{\circ} ; T_{\mathrm{A}} \geqslant 50 \mathrm{~K}\right)$, derived from the contour map shown in Figure $2 \mathrm{~b}$.

'arm' structures are distinctly patchy, a feature not expected on the basis of velocity crowding operative in a smoothly distributed medium in which a smooth spiral gravitational perturbation is present.

The $l, v$ distributions shown in Figures 3, 4, and 5 provide the basis for deriving a line graph, the analog of Figure 1b. We utilize the computer to find maxima from Figure 5. We fix the value of $l$ and find maxima in the $v$-coordinate. The results are displayed in Figure 6. These points have been joined into curves in Figure 7. This last step required human intervention; the computer was instructed which points in Figure 6 to join together to form the observational analog of Figure $1 \mathrm{~b}$. 


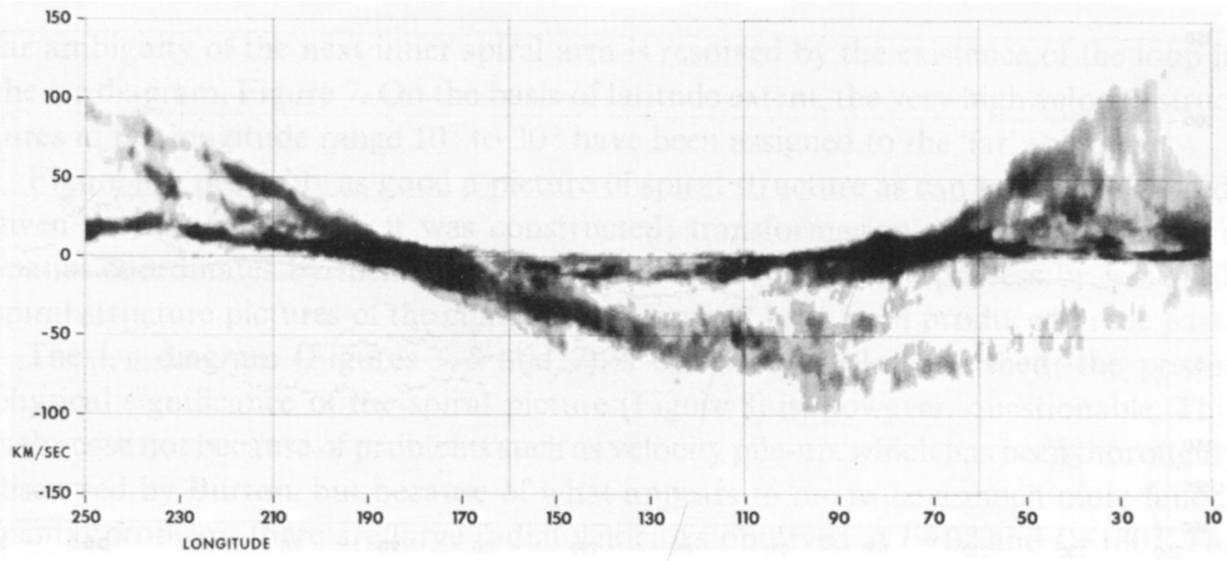

Fig. 3. The observed $l, v$ distribution derived for $T_{\mathrm{c}}=50 \mathrm{~K}$.

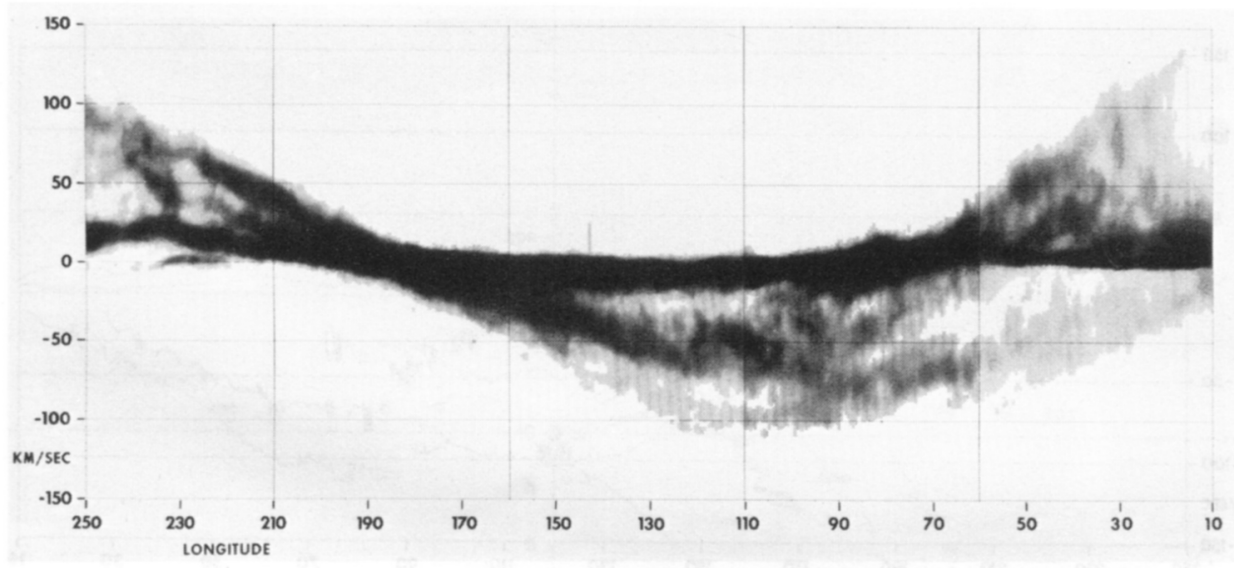

Fig. 4. The observed $l, v$ distribution derived for $T_{\mathrm{c}}=25 \mathrm{~K}$.

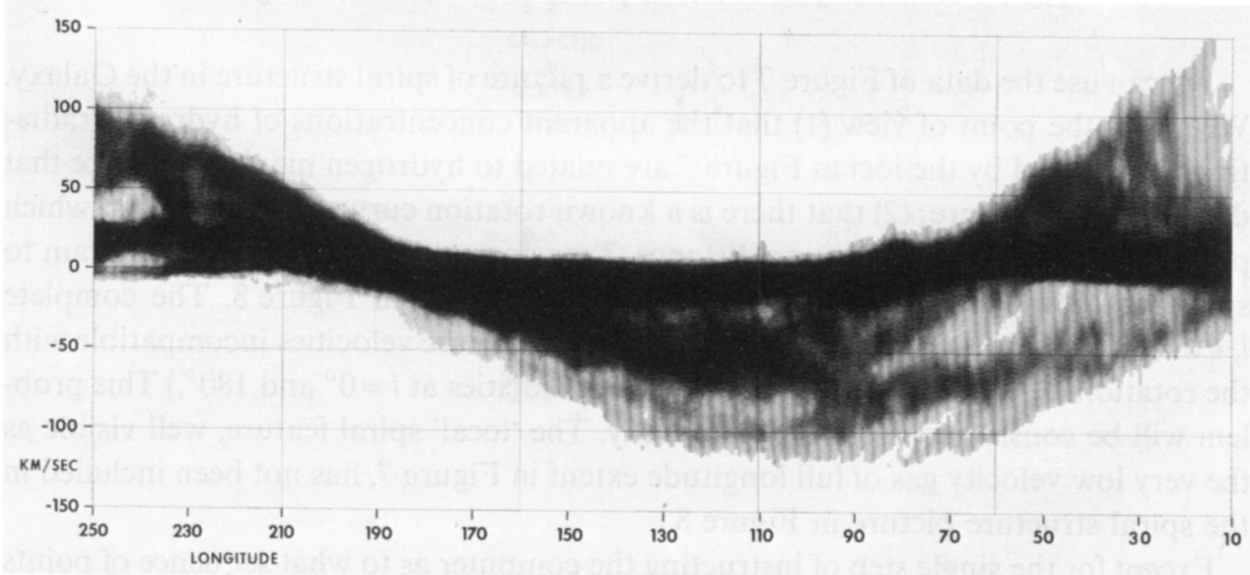

Fig. 5. The observed $l, v$ distribution derived for $T_{\mathrm{c}}=10 \mathrm{~K}$. 


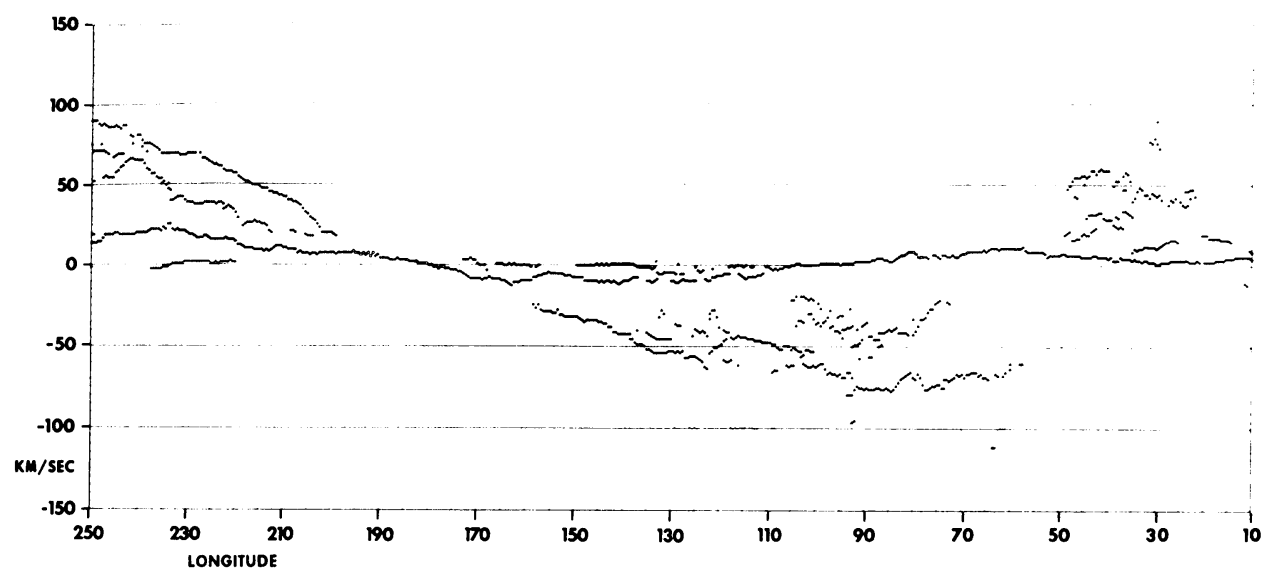

Fig. 6. Maxima in the $l, t$ plane derived from the data of Figure 5.

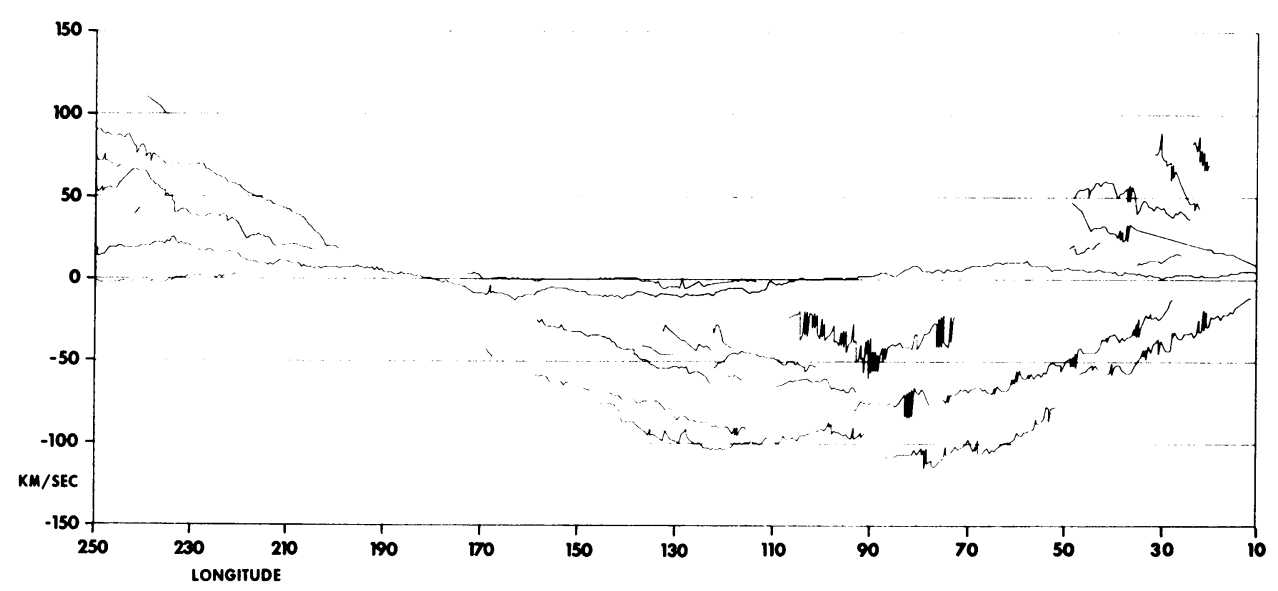

Fig. 7. The $l, v$ diagram formed by joining maxima pictured in Figure 6 .

We can use the data of Figure 7 to derive a picture of spiral structure in the Galaxy. We adopt the point of view (1) that the apparent concentrations of hydrogen radiation represented by the loci in Figure 7 are related to hydrogen maxima in space that define spiral structure; (2) that there is a known rotation curve (Schmidt, 1965) which permits conversion of velocity to distance. Transformation of the $l, v$ line diagram to spatial coordinates produced the spiral pattern shown in Figure 8. The complete $l, v$ diagram in Figure 7 cannot be transformed; there are velocities incompatible with the rotation curve. (Nonzero observed radial velocities at $l=0^{\circ}$ and $180^{\circ}$.) This problem will be considered more fully presently. The 'local' spiral feature, well visible as the very low velocity gas of full longitude extent in Figure 7, has not been included in the spiral structure picture in Figure 8.

Except for the single step of instructing the computer as to what sequence of points is to be joined, the diagram in Figure 8 is completely computer produced. The near- 
far ambiguity of the next inner spiral arm is resolved by the existence of the loop in the $l, v$ diagram, Figure 7 . On the basis of latitude extent, the very high velocity structures in the longitude range $10^{\circ}$ to $30^{\circ}$ have been assigned to the 'far' solution.

Figure 8 is probably as good a picture of spiral structure as can now be produced, given the basis on which it was constructed: transformation of an $l, v$ diagram to spatial coordinates by means of a rotation curve. (This is the process by which all spiral structure pictures of the character of Figure 8 have been produced in the past.)

The $l, v$ diagram (Figures 3-5 and 7) is extremely well established; the precise physical significance of the spiral picture (Figure 8) is, however, questionable. This is the case not because of problems such as velocity pile-up, which has been thoroughly discussed by Burton, but because of what appears to me to be a much more fundamental problem: there are large radial velocities observed at $l=0^{\circ}$ and $l=180^{\circ}$. The well-known 'three $\mathrm{kpc}$ arm' shows a radial velocity of $-53 \mathrm{~km} \mathrm{~s}^{-1}$ at $l=0$; at $l=180^{\circ}$

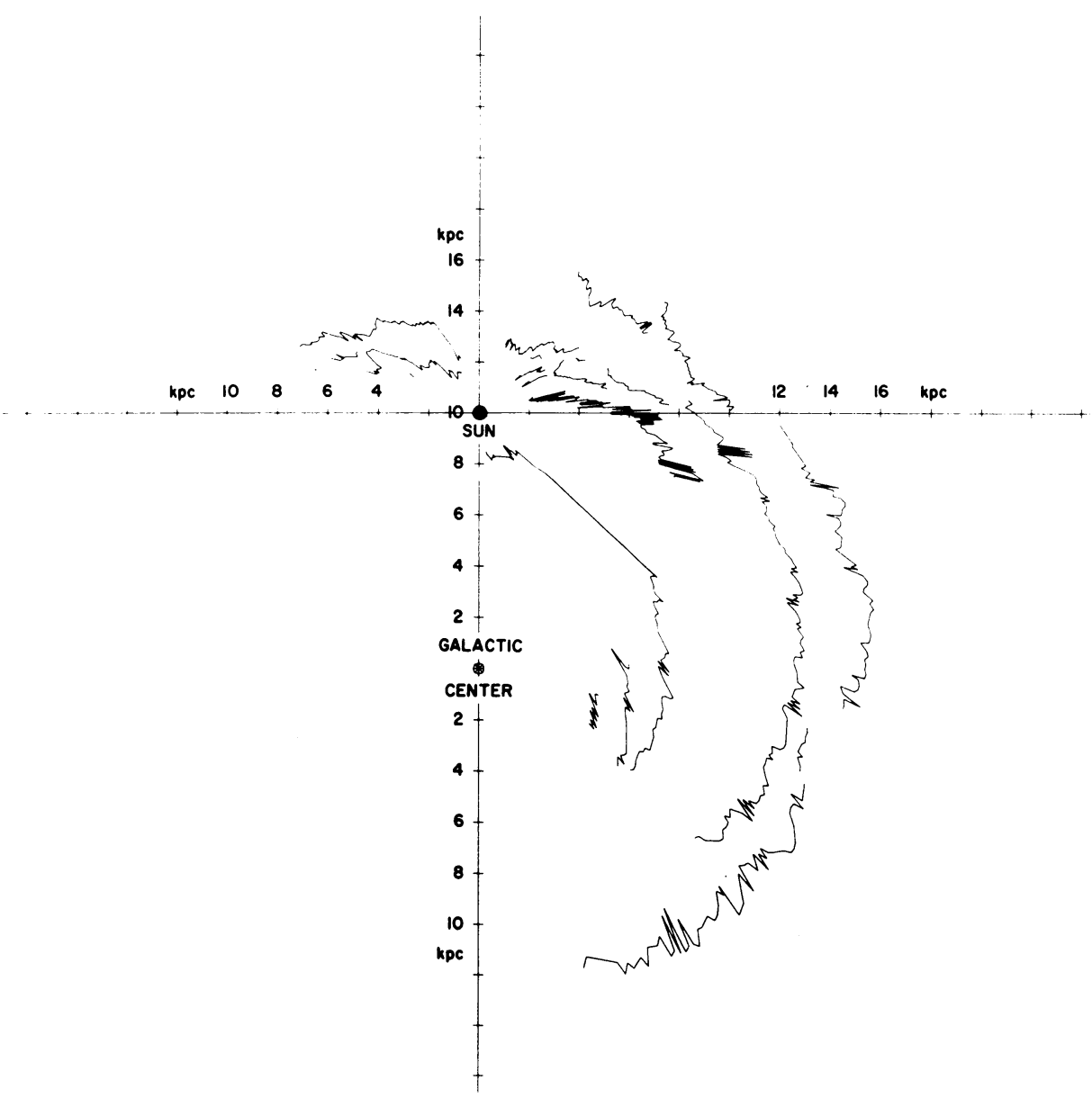

Fig. 8. The spiral pattern derived from the data in Figure 7 with the aid of the Schmidt (1965) rotational model of the Galaxy. 
the outermost spiral arm shown on Figures 5 and 7 exhibits a velocity of $-30 \mathrm{~km} \mathrm{~s}^{-1}$ as found earlier by P. O. Lindblad. It is not possible within the present theoretical framework of galactic dynamics to account for such large velocities. Specifically, they lie outside the range of values encompassed by the current spiral density wave theory. They call into question the whole process currently used to produce pictures of spiral arms by kinematic effects.

Anomalous velocities have been pointed out at $l=0^{\circ}$ and $180^{\circ}$. It is not likely that a velocity anomaly exists only at those specific longitudes. It is probable that the highest velocity spiral feature shown in Figures 5 and 7 in the $l=10^{\circ}$ to $30^{\circ}$ range is directly related to the three $\mathrm{kpc}$ arm. The outermost arm extends over a long range of longitude. There is no velocity discontinuity in that arm near $l=180^{\circ}$, hence there is no reason to expect that the identified velocity anomaly exists only at $180^{\circ}$. The probability is high that radial motions of quite large size exist everywhere along these spiral arms, that distance estimates to these arms, made on the basis of any current dynamical model, are incorrect, and that consequently the picture of spiral structure, derived on the basis of velocity-distance transformation in any current theory, will be in error.

\section{(b) PICTURES ON THE SKY}

The high density of points in the sky coverage of the Berkeley Low Latitude Survey makes it possible to produce pictures of individual spiral features as they appear on the sky in hydrogen radiation. We employ the following technique of production. In Figures 5 and 7 the velocity-longitude locus of the outermost arm of the Galaxy is well shown. Over the longitude range $10^{\circ}$ to $100^{\circ}$ we draw, as a function of longitude, a velocity cut-off that separates this outer feature from adjacent velocity features (Figure 9). At each $l, b$ point observed in the longitude range $10^{\circ}$ to $100^{\circ}$ and latitude range $-10^{\circ}$ to $+10^{\circ}$, we integrate the observed profile over velocity from the velocity cut off (Figure 9) to the greatest negative velocity of the profile. The resultant integral is proportional to the brightness of the outer arm at the $l, b$ point. From the 14661 data points available in the $l, b$-ranges of interest, the pictures shown in Figure 10 were produced. The three pictures, which show the outer arm over the l-range $10^{\circ}$ to $100^{\circ}, b$-range $-10^{\circ}$ to $+10^{\circ}$, are analogous to optical photographs taken on plates of different speeds and contrasts. The spiral arm pictures were produced by using in the computer program three different brightness, blackening relations, the analogs of different HD curves in the case of photographic plates.

Several features of these pictures are noteworthy.

(i) At the ends of the longitude range covered in the pictures we appear to look through one spiral feature to see the outer arm, which, throughout its length in the longitude range covered by this picture, lies high above the galactic plane (the galactic equator is indicated by the white line); it appears flattened on its southern (lower) side.

(ii) Many cloud-like high-z structures lie above the arm. Such features have been observed elsewhere in the galaxy by Kepner (1970). In the case of the outer arm the 


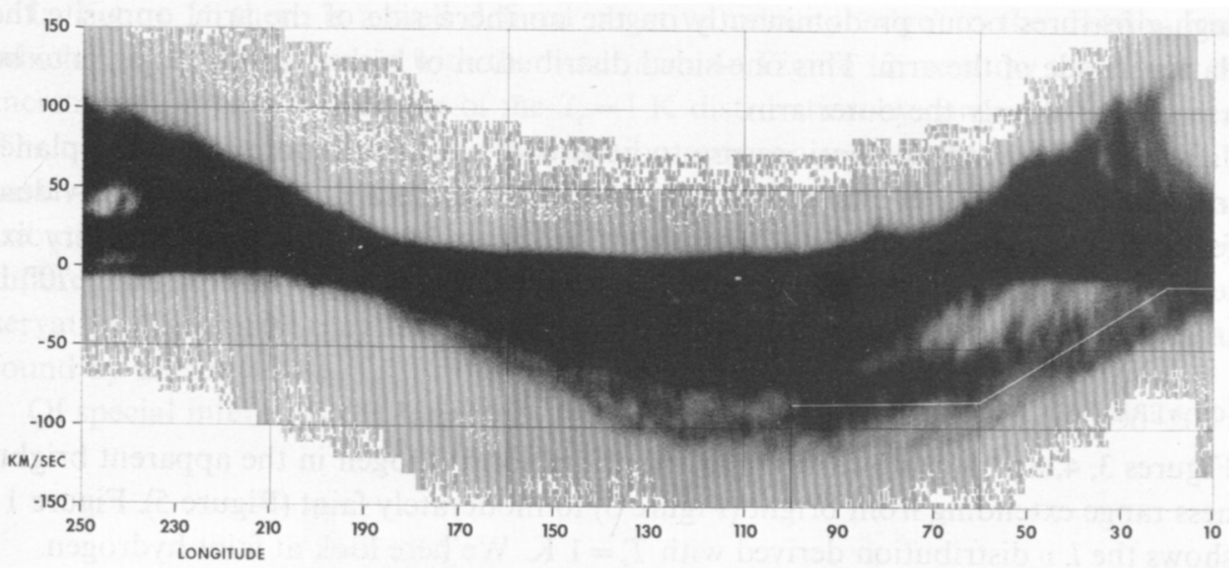

Fig. 9. The $l, v$ distribution shown in this figure was derived with $T_{\mathrm{c}}=1 \mathrm{~K}$. On this distribution has been drawn as polygonal locus the velocity cut-off that separates the outer spiral arm from other velocity features.
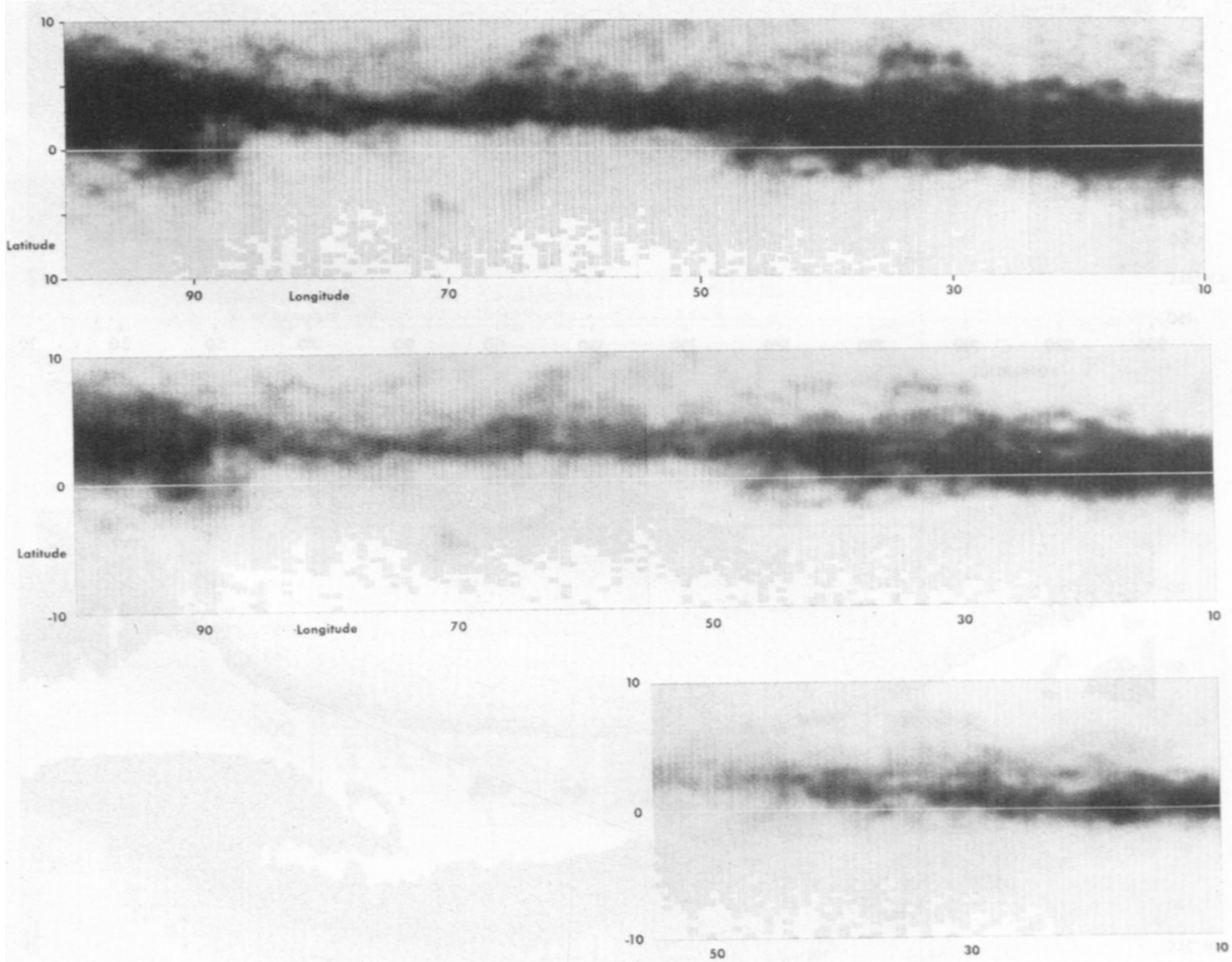

Fig. 10. Pictures of the outer arm of the Galaxy as seen on the sky in the $1420 \mathrm{MHz}$ radiation of neutral hydrogen. The three pictures correspond to optical photographs taken with plates of different speed and contrast. 
high-z features occur predominantly on the northern side of the arm, opposite the flattened side of the arm. This one-sided distribution of high- $z$ objects appears to be a property of only the outer arm.

(iii) The cloud-like extensions rise to heights of a $\mathrm{kpc}$ and more above the plane; in some instances they are of 1-2 kpc length along the arm. Masses of individual features reach values $>10^{6} M_{\odot}$; many are in excess of $10^{5} M_{\odot}$. The faint very extensive arc of gas in the negative latitude range at approximate longitude $70^{\circ}$ is under detailed investigation.

(c) VERY FAINT FEATURES IN THE GALACTIC HYDROGEN

Figures 3, 4, and 5 exhibit the $l, v$ distributions for hydrogen in the apparent brightness range extending from bright (Figure 3) to moderately faint (Figure 5). Figure 11 shows the $l, v$ distribution derived with $T_{\mathrm{c}}=1 \mathrm{~K}$. We here look at faint hydrogen.

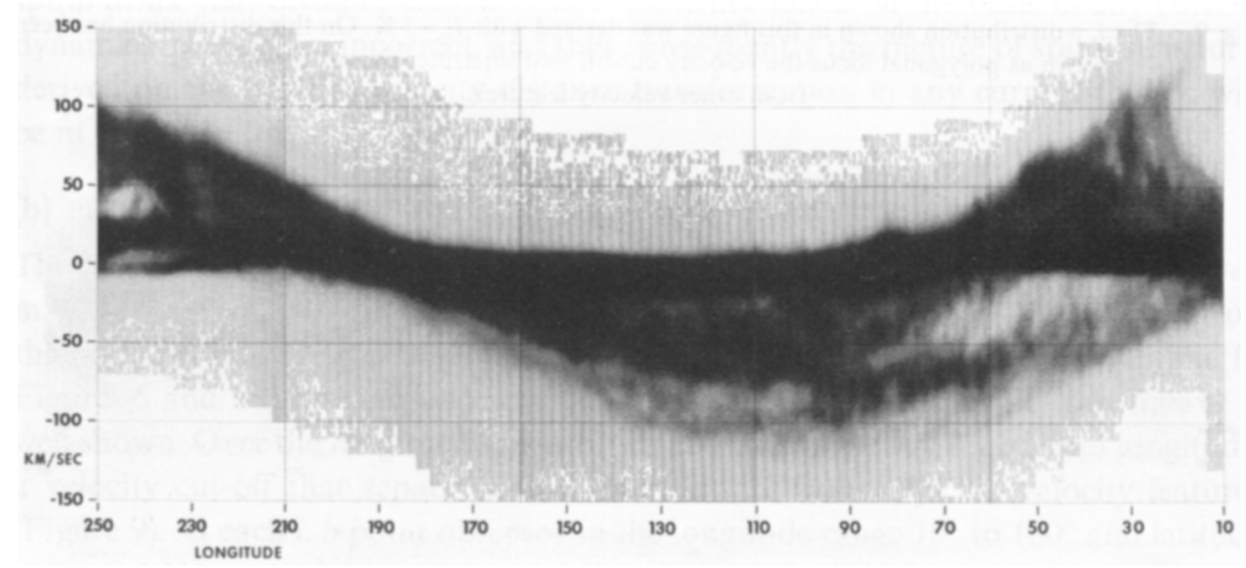

Fig. 11. The $l, v$ distribution derived for $T_{\mathrm{c}}=1 \mathrm{~K}$.

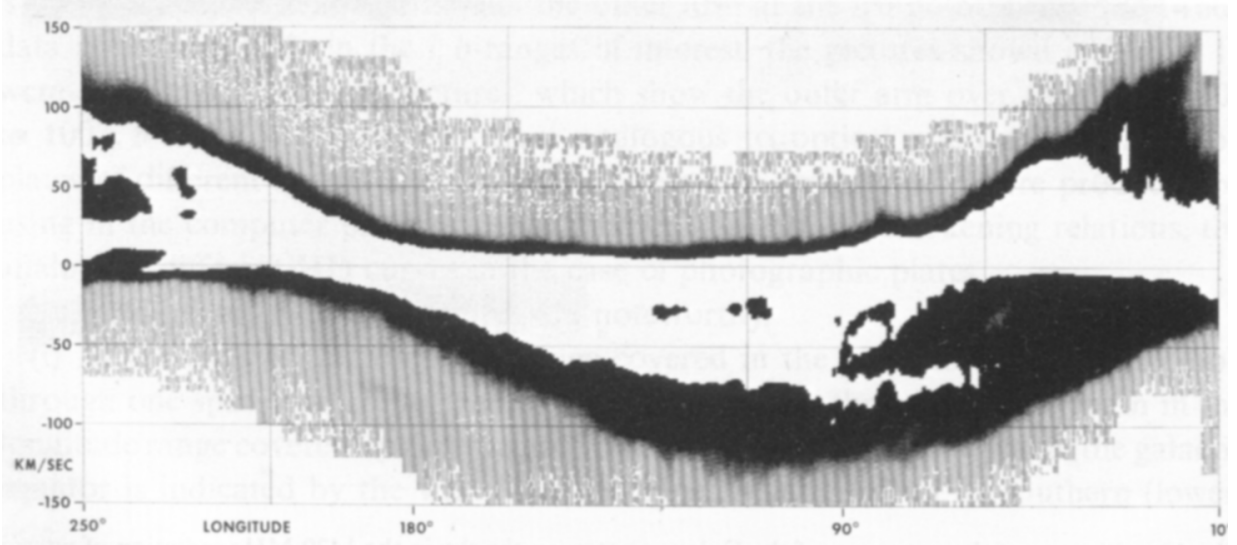

Fig. 12. The $l, v$ distribution derived for $T_{\mathrm{c}}=1 \mathrm{~K}$. The contrast has been increased over that shown in Figure 11 by computer techniques. 
In Figure 11 there is a well defined negative velocity 'edge' to the distribution, which ends rather abruptly with the outer arm. In Figure 12 we show the results of increasing the picture contrast of the $T_{\mathrm{c}}=1 \mathrm{~K}$ distribution by computer techniques. The negative velocity edge of the distribution remains quite sharp; only a few weak tassel-like extensions appear predominantly on the negative velocity side of the distribution. Many of these are of such longitude extent that they are composed of many different observations made at widely different times. They do not appear to be observational artifacts; they show strong correlation with negative velocity extensions found by Dieter (1971).

Of special interest is the long negative extension in the longitude range $190-200^{\circ}$,

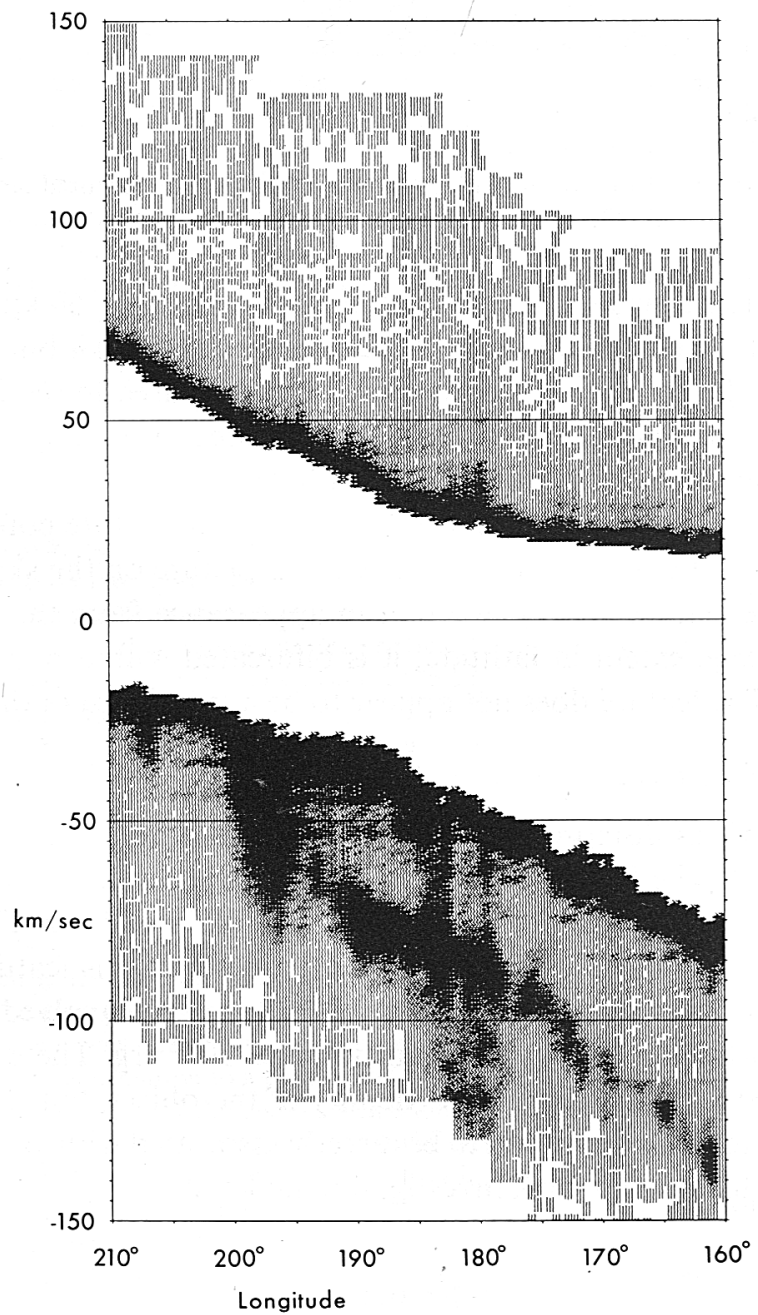

Fig. 13. The $l, v$ distribution derived for $T_{\mathrm{c}}=1 \mathrm{~K}$ for a section of the galaxy. Contrast in this picture is very high, exceeding that of Figure 12. 


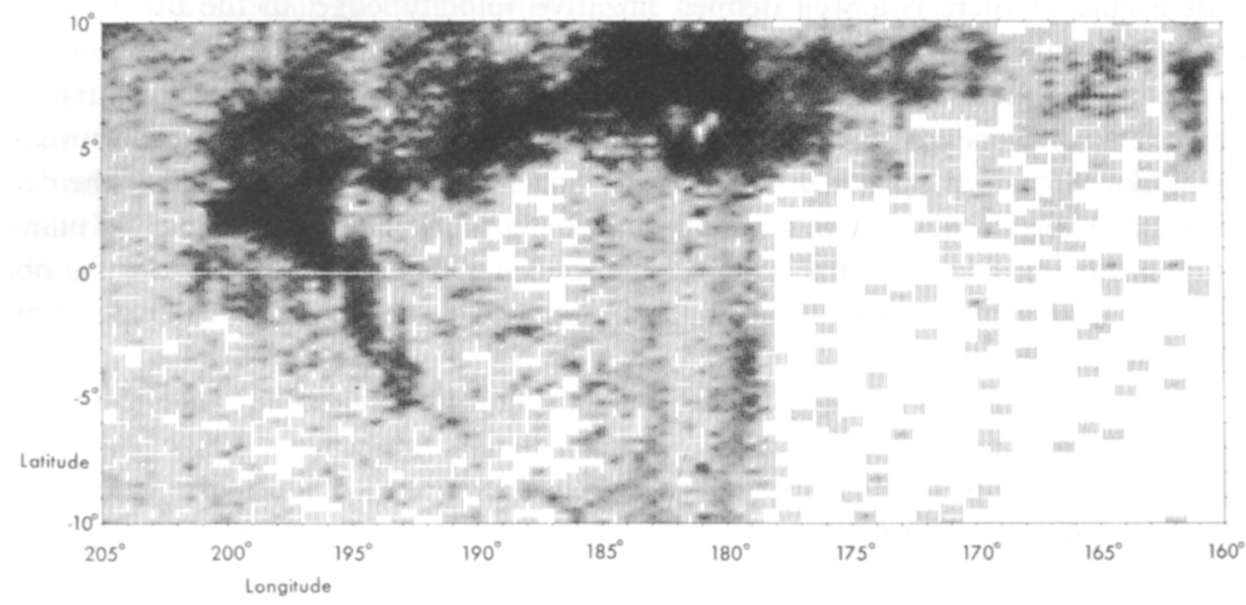

Fig. 14. Computer-produced picture of the feature shown in Figure 13 as it would appear on the sky in the $1420 \mathrm{MHz}$ radiation of neutral hydrogen.

and the weak feature showing a velocity run extending from $-50 \mathrm{~km} \mathrm{~s}^{-1}$ to -100 $\mathrm{km} \mathrm{s}^{-1}$ in the longitude range $200^{\circ}$ to $160^{\circ}$. These features are exhibited more clearly in Figure 13, where the contrast has once more been increased in the computer. The jet-like extension in the region $l=200^{\circ}$ has been discussed previously (Weaver, 1970). The feature showing the velocity run with longitude has been identified as a spiral arm by Verschuur (1973). A picture of the gas producing the feature pointed out above and identified by Verschuur as an arm is shown as a picture on the sky in Figure 14. The structure of the feature is very different in appearance from the arm shown in Figure 10; it is of large extent in latitude; it is bifurcated with a weak extension to negative latitudes. The feature does not appear to be a spiral arm of the Galaxy.

\section{(d) INTERESTING OBJECTS}

The Berkeley Survey has disclosed many jets, holes and other types of structures of astrophysical interest. The feature located at $l=196^{\circ}$ and discussed earlier provides an example of a large jet. An example of an extensive hole is visible in Figures 4 and 5 at longitude $80^{\circ}$ and velocity $-77 \mathrm{~km} \mathrm{~s}^{-1}$. The diameter of this feature is approximately $1.5 \mathrm{kpc}$. The expansion velocity is $10 \mathrm{~km} \mathrm{~s}^{-1}$. The mass involved is of the order several $\times 10^{6} M_{\odot}$, while the energy of expansion is $\sim 10^{52} \mathrm{erg}$. There are a number of supernova remnants in the immediate vicinity of the object, but these appear to possess rather small angular diameters to be directly associated with, or the immediate cause of, the hole, which we are now investigating in detail.

\section{References}

Dieter, N. H.: 1971, Astron. Astrophys. 12, 59

Kepner, M.: 1970, Astron. Astrophys. 5, 444. 
Schmidt, M.: 1965, Stars and Stellar Systems 5, 528.

Verschuur, G.: 1973, Astron. Astrophys. 22, 139.

Weaver, H.: 1970, IAU Symp. 39, 22 (see p. 48 especially).

Weaver, H. and Williams, D. R. W.: 1973, Astron. Astrophys. Suppl. 8, 1.

\author{
Harold Weaver \\ Radio Astronomy Laboratory, \\ University of California, \\ Berkeley, Calif. 94720, U.S.A.
}

\title{
DISCUSSION
}

Mathewson: What is your model for the perturbations in the velocity field of the local gas?

Weaver: This model is similar to the one suggested by Wesselius and Fejes. If there is some energetic event that interrupts the circular motion of the gas the material will start in an elliptical orbit. If it has less angular momentum than the Sun we run into it. From our position it is like a jet stream coming over our heads at a distance of around $70 \mathrm{pc}$. It shows negative velocities at $l \sim 110^{\circ}-120^{\circ}$ and positive velocities $180^{\circ}$ further around in longitude but at the same latitude. The mass is not large $\left(\sim 2 \times 10^{3} M_{\odot}\right)$.

Mezger: When you make the transition from velocity space to spiral structure a spiral arm emerges inside the solar circle. I was very glad to see this feature, since usually one sees on maps of spiral structure derived from $21-\mathrm{cm}$ observations inside the solar circle features which reflect the author's best guess rather than hard observational facts. On the other hand, we know that giant $\mathrm{H}$ il regions disappear at distances of about $12 \mathrm{kpc}$ from the galactic center. Therefore, if we limit interpretations of $21-\mathrm{cm}$ observations to features outside the solar circle we may actually miss the major spiral structure of our Galaxy. Now, my question is, how did you manage to resolve the distance ambiguity for features located inside the solar circle?

Weaver: There are only two positions there where there is any distance ambiguity. One of them is resolved by the loop in the $l, v$ diagram which automatically tells which is near and which is far. It also has a vastly different latitude extent on the two sides of the loop. It is very easy to resolve. If there were a lot of spiral features in the interior as seen in the hydrogen, I think there might be problems. But there is not a large amount of structure that appears in this organized way in the interior of the Galaxy.

Mezger: Do you tell us the spiral arms also are tilted?

Weaver: They are tilted only $8^{\circ}$.

Simonson: It is important to make models that contain the velocity field for comparison with the observed $T_{\mathrm{b}}(l, v)$ diagram. Mapping the peak intensities in the profiles may not really trace the density maxima.

Weaver: I am in complete agreement. If we wish to obtain a 'precise' picture of the spiral structure of the Galaxy, we certainly must have a 'precise' model of the velocity field and we must transform the $T\left(l, v \mid T_{\mathrm{A}} \geqslant T_{\mathrm{c}}\right)$ distribution. As I tried to emphasize in my remarks, however, the spiral picture I presented was not meant to be such a 'precise' picture. It was derived by the same techniques used to derive the spiral pictures published in the literature during the past several years and was meant to be comparable with those pictures. The main purpose of the part of my discussion dealing with the spiral picture was to emphasize that while we can now derive a very precise and beautiful $T\left(l, v \mid T_{\mathrm{A}} \geqslant T_{\mathrm{c}}\right)$ distribution, we cannot, I believe, make the transformation to a precise and beautiful distribution in space. I believe that we do not yet understand the dynamics of the Galaxy well enough to derive the velocity field required to make the transformation. The feature of the $T\left(l, v \mid T_{\mathrm{A}} \geqslant T_{\mathrm{c}}\right)$ distribution that convinces me of our inadequate knowledge is the very large radial motions we observe in the gas.

Simonson: Would you care to model the $-70 \mathrm{~km} \mathrm{~s}^{-1}$ motion at $l=180^{\circ}$ with a dispersion ring as for the $-53 \mathrm{~km} \mathrm{~s}^{-1}$ motion at $l=0^{\circ}$ ?

Weaver: No, I would not care to do so. In my view the $-70 \mathrm{~km} \mathrm{~s}^{-1}$ feature at $l=180^{\circ}$ is not an arm of the Galaxy.

Van Woerden: I admire Weaver's computerized and photographic techniques for reduction and display of data. However, some of the computer-defined ridges of maxima of $N_{\mathbf{H}}$ in the $(l, v)$ plane should not be 
interpreted as density maxima in the galactic $(L, R)$ plane, but rather as effects of long line of sight and/or velocity crowding - as Burton has clearly demonstrated. But this should not make us unduly pessimistic. Burton's review shows that, with careful analysis and due regard for dynamical considerations, progress in understanding our Galaxy is possible.

Weaver: Ridges will occur if there is velocity pile-up, ridges will occur if there is contrast between arm and interarm densities. The computer technique will pick out ridges, there's no question about that; but that was not saying what makes the ridges. That I think is the part that I am more and more pessimistic about. I can see how to make excellent pictures in the observational space, but I do not see how at the present time to produce a convincing picture of the spatial distribution of gas. I lose my optimism because of these large velocities which I think are not present in the theory. 